\title{
The effect of confinement on the brittle compressive fracture of ice
}

\author{
E. M. Schulson, D. E. Jones And G. A. Kuehn \\ Thayer School of Engineering, Dartmouth College, Hanover, NH 03755, U.S.A.
}

\begin{abstract}
Granular, fresh-water ice was deformed at $-40^{\circ} \mathrm{C}$ at $10^{-3} \mathrm{~s}^{-1}$ under confinement. Loading was applied such that confining stress, $\sigma_{2}$ and $\sigma_{3}$, were equal to each other and proportional to the highest stress, $\sigma_{1}$; i.e. $\sigma_{2}=\sigma_{3}=R \sigma_{1}$. Two regimes were evident. At lower confinement, the fracture stress, $\sigma_{1 \mathrm{f}}$, increases markedly as $R$ increases. At higher confinement, $\sigma_{1 \mathrm{f}}$ increases less sharply. The transition occurs at about $R=0.15$. Wing cracks were seen in the ice fractured within the low- $R$ regime. The data are analyzed in terms of the frictional crack sliding-wing crack mechanism of brittle compressive fracture.
\end{abstract}

\section{INTRODUCTION}

When an ice feature driven by wind and ocean currents contacts an offshore platform, an ice floe or some other obstacle, pressure develops within the contact zone. If large enough, it can fracture the ice. The question is: how large is large enough? The answer depends on a number of factors, including the temperature and velocity of the feature as well as its salinity and microstructure. It depends also on the stress state. Owing to the confining influence of the material outside the zone, the material within is acted upon by stresses both orthogonal to and parallel to the direction of incidence. This confinement effectively "holds the material together" and thereby raises the pressure to cause failure above the uniaxial fracture stress. How much above depends on the degree of confinement, which varies with position and boundary conditions, and on the failure mechanism.

This paper considers the effect of confinement on the brittle compressive fracture of granular, fresh-water ice. Specifically, it presents preliminary results from experiments performed at $-40^{\circ} \mathrm{C}$ on material confined through proportional loading, and reveals two distinct regimes of behavior. Also, the paper presents an analysis based upon the frictional crack sliding-wing crack mechanism of compressive fracture. The work is part of a larger study which will be published in due course.

\section{EXPERIMENTAL WORK}

The ice was prepared in the laboratory using a variation on the method described by Cole (1979). "Seeds" of one size $(9.5-11.2 \mathrm{~mm})$ were obtained by crushing and sieving bubble-free sheets of fresh-water ice, and then charged into a cube-shaped mold of edge length $158 \mathrm{~mm}$. Distilled, deionized and degassed water was added and the ice-water mixture was frozen inward by passing coolant at $-5^{\circ} \mathrm{C}$ through cooling coils attached to the sides of the mold. Water was passed slowly through the mixture during freezing to reduce freezing strains and to permit the release of undissolved gases. The product was relatively bubble free, albeit slightly hazy in appearance, and consisted of equi-axed and randomly oriented grains of $7.3 \mathrm{~mm}$ size. (Grain-size was obtained from the method of linear intercepts and corresponds to the average spacing between grain boundaries on a thin section.) Cubes $155 \mathrm{~mm}$ on edge were machined from the ingots using a milling machine. The dimensions were within $\pm 0.07 \mathrm{~mm}$ and the orthogonality between the faces was at least as good as could be detected using a high-precision machinist's square.

The cubes were deformed under conditions where the ice was brittle. The principal variable was the degree of confinement, $R$, which ranged from $0<R<0.3$. $R$ is defined as the ratio of the average confining stress, $\left(\sigma_{2}+\right.$ $\left.\sigma_{3}\right) / 2$, to the maximum stress, $\sigma_{1}$, where $\sigma_{1}, \sigma_{2}$ and $\sigma_{3}$ are the three principal stresses. In the present experiment, $\sigma_{2}=\sigma_{3}$ and proportional loading was applied; i.e. $\sigma_{2}=$ $\sigma_{3}=R \sigma_{1}$.

The specimens were loaded using a true multi-axial MTS testing system (MATS). MATS is a servo-hydraulic system which was specially designed and built by MTS for the Ice Research Laboratory of Thayer School of Engineering, Dartmouth College. It consists of six actuators capable of applying loads up to $1 \mathrm{MN}$ in each of three mutually perpendicular directions at rates up to $10 \mathrm{~mm} \mathrm{~s}^{-1}$, without altering the position of the centroid of the test specimen. The system is housed within a cold room which can reach below $-40^{\circ} \mathrm{C}$ and hold temperature to within $0.2^{\circ} \mathrm{C}$. The loads were transmitted through brush-type metallic platens which are described by Gies (1988); they are similar to but larger than the ones used by Hausler (1981) in his multi-axial system. Three pairs of MTS displacement gauges were mounted across diagonally opposing sets of bristles once the ice was positioned within the system. In this way, the average displacement in each direction was obtained. The 
experiments were performed at $-40^{\circ} \mathrm{C}$ under displacement control, which was set to maintain a strain rate of $10^{-3} \mathrm{~s}^{-1}$ along the direction of greatest load. Post-test analysis revealed that the rate remained constant during each test but that the actual rates varied between 0.7 and $1.2 \times 10^{-3} \mathrm{~s}^{-1}$. The loads giving $\sigma_{2}$ and $\sigma_{3}$ were slaved to the load giving $\sigma_{1}$. Data were collected using a 30 channel, computer-based system.

Boundary conditions are an important factor when considering brittle behavior of ice (Schulson and others, 1989). The brushes, the performance of which is described briefly by Lasonde and others (1988) and by Schulson and others (1989), were designed to be stiff longitudinally, but compliant transversely, in order to reduce end effects. They are not perfect, but to the best of our knowledge, they appear to exert little influence on either the fracture stress or the fracture mode.

\section{RESULTS}

Twelve experiments were performed. In each case the ice deformed in a brittle manner. The loads rose essentially linearly with time and then fell sharply when the specimen failed. Under lower confinement $(R \leq 0.2)$, the loads subsequently rose and fell again, in a saw-tooth manner, but to levels less than half of that reached at failure. Curiously, both the period and the amplitude of this "stick-slip" kind of deformation increased with increasing confinement. Under higher confinement, on the other hand, the post-failure loads were approximately steady at around half the failure loads. Figure 1 shows typical load-time curves. The ends denote the point where the tests were terminated and not where some other physical process occurred.

For the present purposes only the failure loads were examined. These revealed two regimes (Fig. 2). For $R \lesssim 0.15$ the failure stress, $\sigma_{1 \mathrm{f}}$, increases markedly with moderate confinement. For instance, the failure stress of ice confined at $R=0.1$ is about three times that of unconfined material. For $R \geq 0.15$, on the other hand, $\sigma_{1 \mathrm{f}}$ still increases with confinement, but the dependence is less.

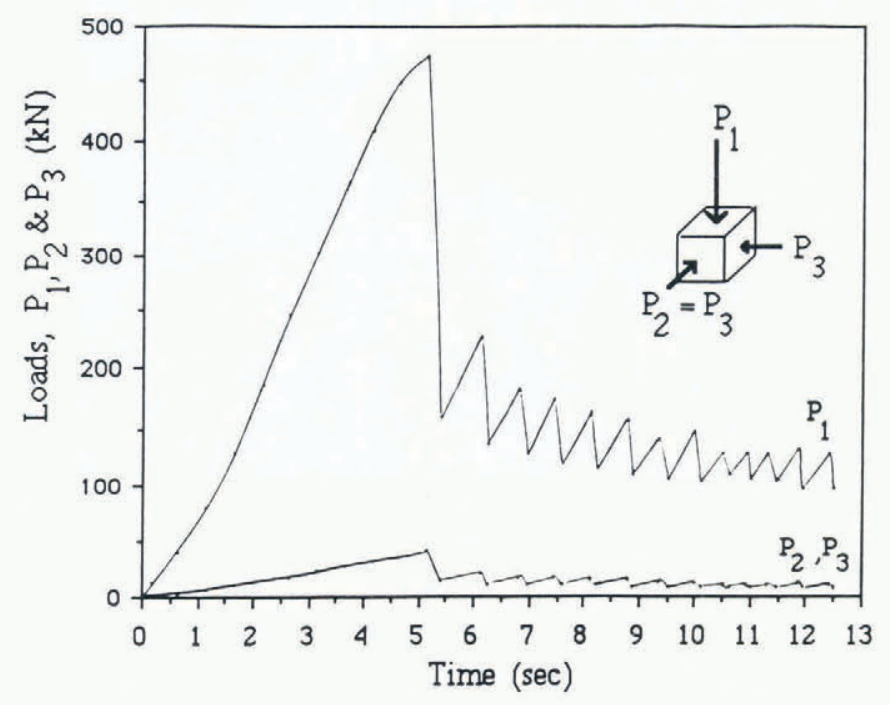

Fig. 1. Load versus time curves for $R=0.075$.

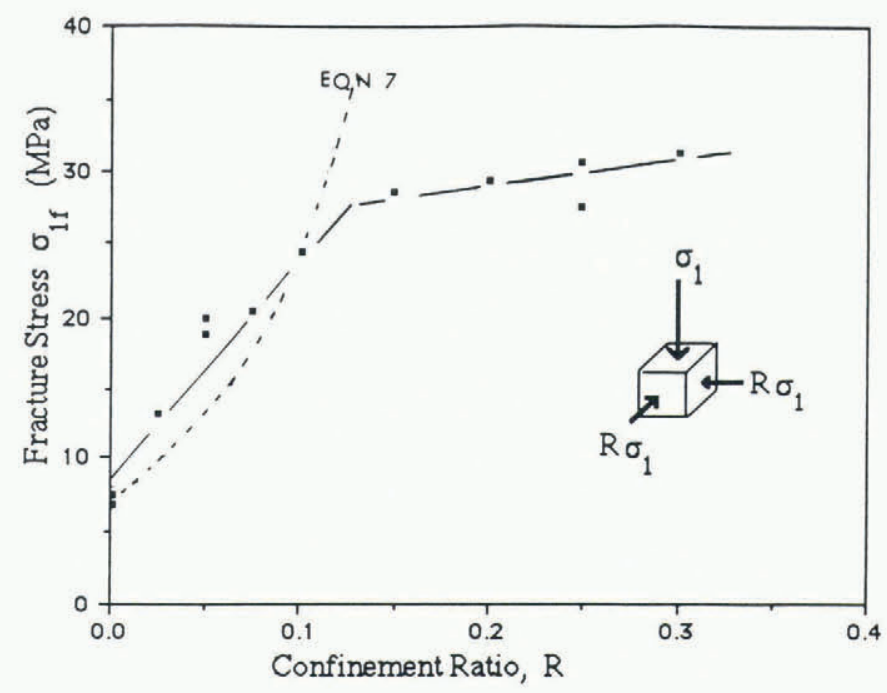

Fig. 2. Brittle compressive strength versus confining ratio, $R$. The broken curve is a plot of Equation (7).

The unconfined strength, incidentally, is about $40 \%$ lower than the strength of similar material tested as cylinders to which Synthane end caps had been bonded (Schulson, 1990). About half of this difference is a reflection of the lower degree of constraint afforded by the brushes, as noted earlier (Schulson, 1990). The other part may be due to differences in material (the present ice was somewhat cloudier) and in specimen shape.

Whereas a distinction between two brittle regimes in ice has not been made previously, it could be argued that evidence for such a distinction is embodied within the data of Jones (1982). From results obtained using a conventional triaxial cell, he showed that the compressive strength of finely grained $(d=0.8 \mathrm{~mm})$, fresh-water ice, deformed at $-11^{\circ} \mathrm{C}$ at strain rates both comparable to and higher than that employed here, increases markedly with low levels of confinement, but only slightly with higher levels. (Note that he plotted as strength the maximum minus the minimum stress.) On the other hand, when it is remembered that Jones observed essentially ductile behavior in all but his unconfined specimens, in keeping with their finer grain-size and with the higher test temperature, comparison with the present study may be misleading.

The unconfined ice failed by macroscopic splitting along planes parallel to the direction of highest compressive stress. The confined material exhibited both splits and macroscopic shear faults, the splits again being parallel to the direction of highest compressive stress.

Thin sections revealed two other features. Wing cracks, or out-of-plane extensions on inclined cracks, were seen in specimens deformed under low confinement (Fig. 3). The inclined or parent crack had nucleated at a grain boundary. This is evident upon comparing the images obtained under non-polarized illumination with those obtained through cross-polarized filters. The wings had then propagated transgranularly in a direction parallel to the highest compressive stress. The wing cracks are significant because they provide direct support for the operation of a classical mechanism of brittle compres- 

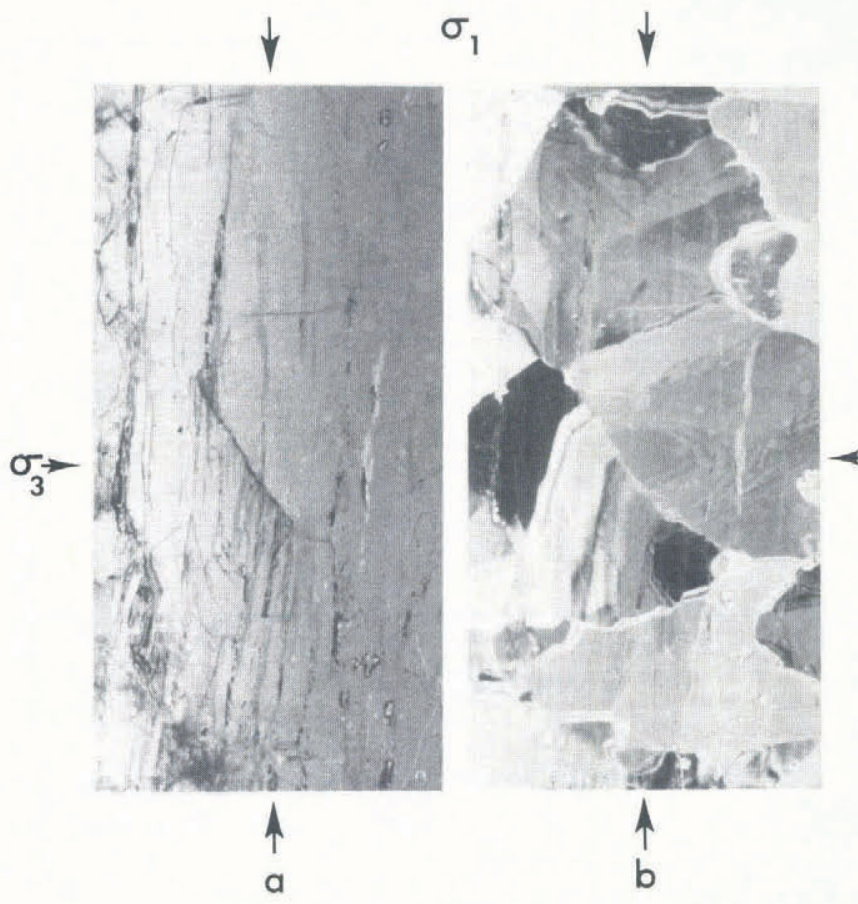

Fig. 3. Thin sections from a fractured specimen which failed via splitting $(R=0.025)$ showing a wing crack (magnification $=2.5 \times$ ); (a) non-polarized image, (b) cross-polarized image. Note that the inclined crack nucleated along a grain boundary, whereas the wings propagated transgranularly.

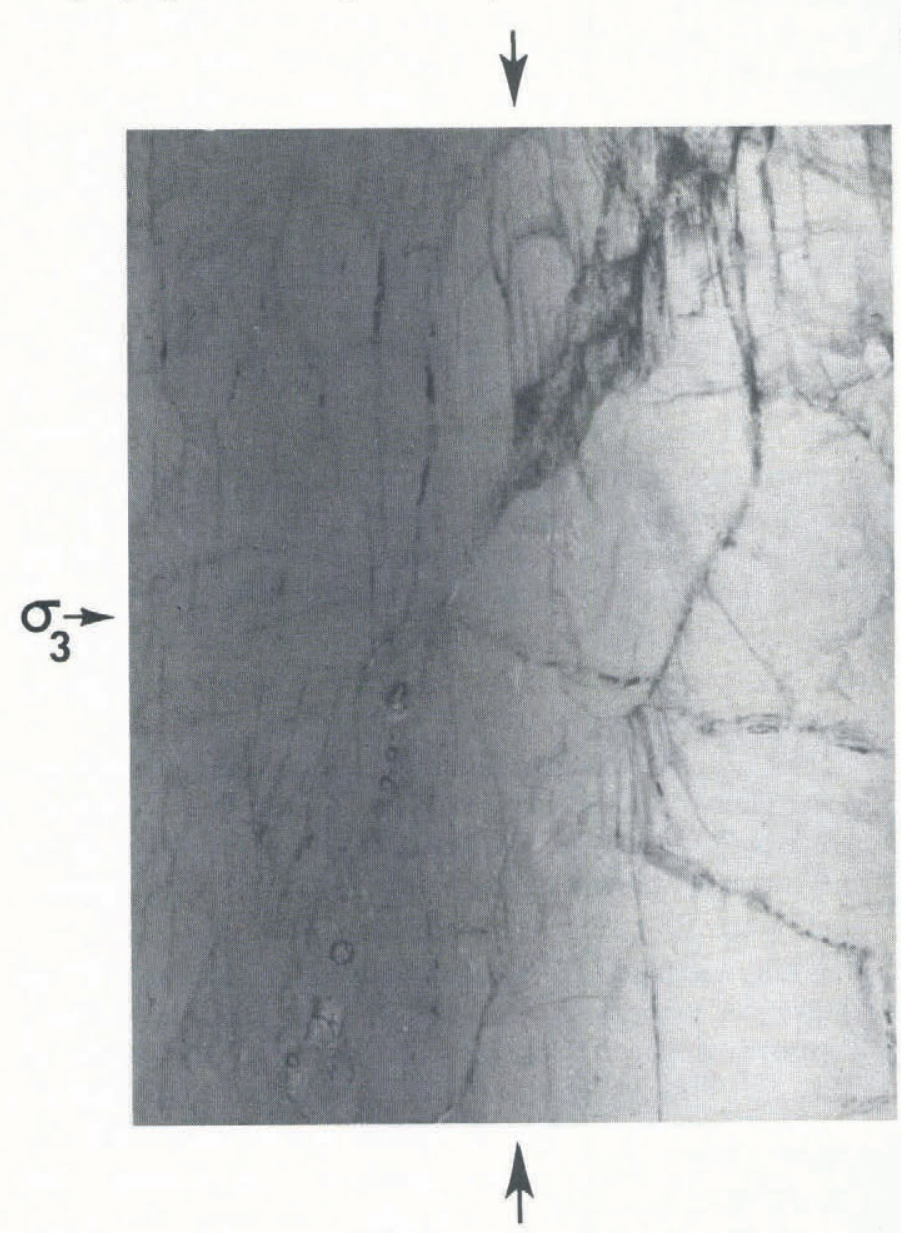

a sive fracture; viz. the frictional crack sliding mechanism (Griffith, 1924; McClintock and Walsh, 1962; NematNasser and Horii, 1982; Ashby and Hallam, 1986). The mechanism is invoked in the analysis below. The other feature is granulation within the shear fault and the attendant offset (Fig. 4). The scale of the granules is difficult to specify, but is certainly finer than the initial grainsize and would appear to be of the order $1 \mathrm{~mm}$ or less. The granules appeared to be tightly packed but to lack cohesion. Granulation is significant because it suggests that the mechanics of fracture under higher confinement may be governed by the flow of granular media.

\section{DISCUSSION}

Attention now turns to failure within the regime of lower confinement. The issues to be discussed are the marked sensitivity of the failure stress to the degree of confinement and the transition from one regime to the other.

Consider the frictional crack sliding-wing crack mechanism (Fig. 5). For the purpose of illustration, the case of plane strain is depicted with through-thickness cracks. Although this is not the situation in the present experiments, it helps to convey the mechanics. Within material which is initially free from cracks, as in the present experiments, the inclined cracks nucleate during loading at stresses well below the failure stress (Kalifa and others, 1989; Schulson and others, 1989; Cannon and others, 1990). In the approximately dozen cases to date

\section{$\sigma$}
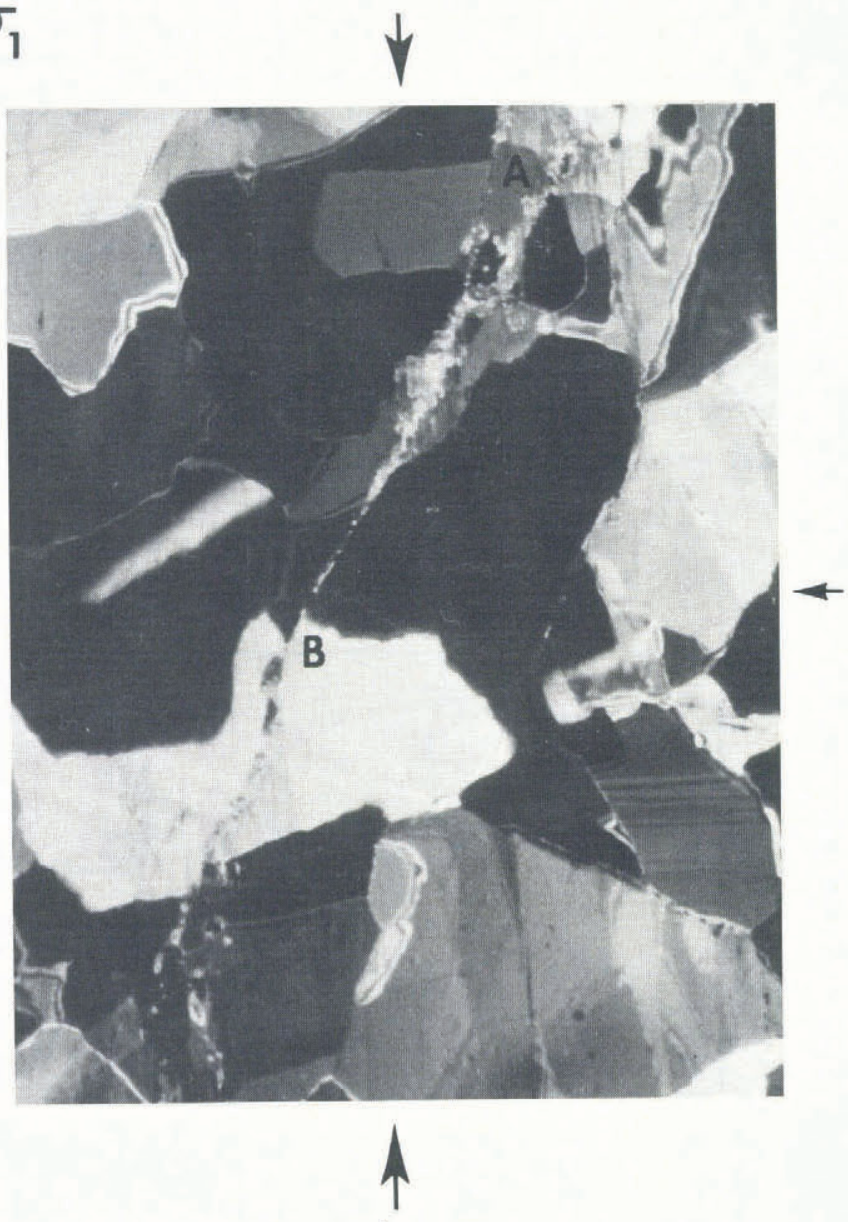

b

Fig. 4. Thin section from a fractured specimen showing a granulated, shear band $(R=0.15$, magnification=2.5 $):($ a) non-polarized image, (b) cross-polarized image. Note the offset along the fault at $A$ and $B$ in (b). 

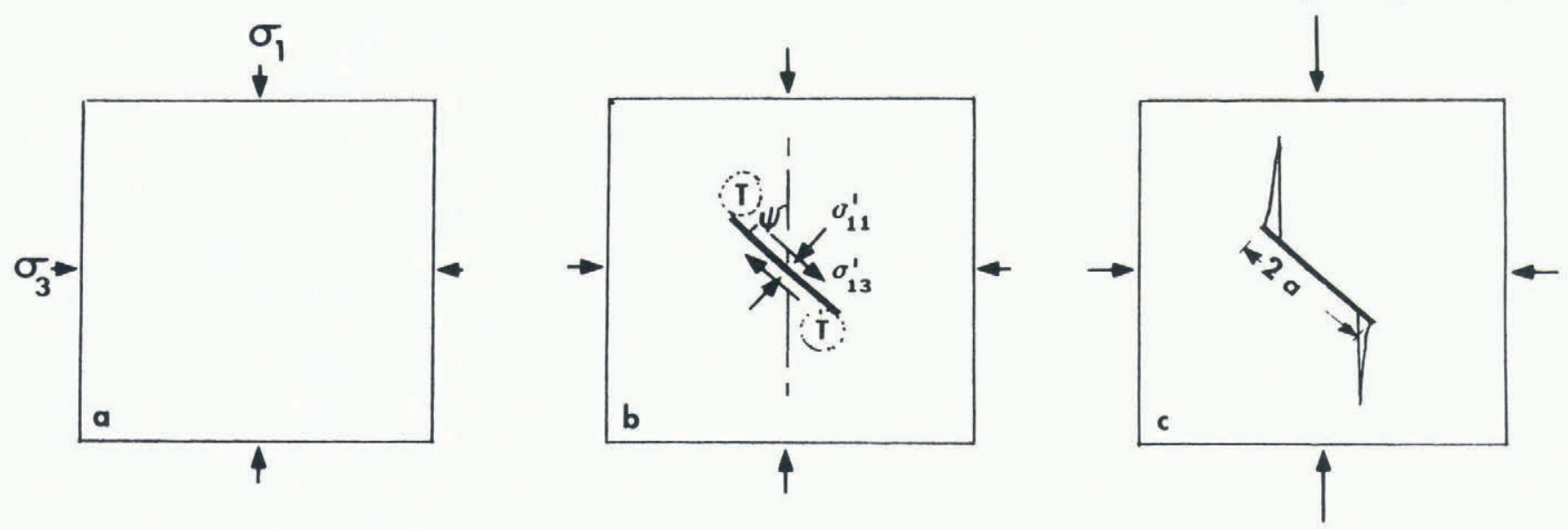

Fig. 5. Schematic sketch of the frictional crack sliding-wing crack mechanism: (a) low stress: no cracks, (b) intermediate stress: inclined crack, (c) high stress: wings initiated and lengthened.

in which wing cracks have been seen in ice, the parent cracks were inclined by about $\psi=45^{\circ}$ to the direction of highest loading. Shear tractions, $\sigma_{13}^{\prime}$, act across the opposing faces and tend to cause sliding. (Primed terms are the components of the stress tensor expressed with respect to an orthogonal coordinate system rotated $\psi$ degrees with respect to the principal coordinate system.) Resisting this tendency is a frictional stress which is the product of the stress normal to the plane of the crack, $\sigma_{11}^{\prime}$, and the friction coefficient, $\mu$. The net shear stress, $\tau$, driving sliding is

$$
\tau=\sigma_{13}^{\prime}-\mu \sigma_{11}^{\prime}
$$

(All compressive stresses are taken here as positive.) When $\tau$ reaches a critical value, wings nucleate within the tensile zones ( $\mathrm{T}$ in Fig. 5). With increasing load they lengthen in a stable manner via mode-I growth. This occurs when the mode-I stress intensity factor at the wing tips reaches the critical stress intensity factor for crack propagation; i.e. when $K_{\mathrm{I}}=K_{\mathrm{IC}}$. Eventually, the wings can become long enough to split the material, either through a single crack running the full length or, more likely, through the linking up of two or more cracks. If the confinement impedes growth, the wings may link to form a shear fault (Argon and others, 1983).

Confinement reduces $\tau$ and, thus, the tendency for the inclined cracks to slide under a given principal stress $\sigma_{1}$. This reduction has two effects. The first is to raise the magnitude of $\sigma_{1}$ for the wings to reach a specific length. By implication, this effect increases the failure stress, provided that failure corresponds to a critical wing length. The second effect occurs when $\tau$ is greatly reduced (say to $\tau \approx 0$ ), and that is to suppress the cracksliding mechanism altogether. Inclined cracks will continue to nucleate, but will resist the initiation and growth of wings. Instead, the crack density will increase until it reaches a critical level, at which point localized granulation and shear faulting occur. The faulting mechanism is still obscure.

The above description may be quantified as follows, with reference to the coordinate systems in Figure 5. The analysis is a derivative of the treatment by Ashby and Hallam (1986). The traction, $\tau$, effective in causing sliding can be shown to be given by the relationship

$$
\tau=\sigma_{1}\left\{(1-R)\left(\sin \psi \cos \psi-\mu \sin ^{2} \psi\right)-R \mu\right\},
$$

where the other parameters are defined above and all normal stresses are positive. Equation (2) and the equations below, in which the term $(1-R)$ appears, are limited to the range $0 \leq R<R_{0}$ where $R_{0}$ (defined below) is the degree of confinement at which $\tau=0$. The traction is independent of the orientation of the crack with respect to directions $X_{2}$ and $X_{3}$, because $\sigma_{2}=\sigma_{3}$. As noted, $\psi=45^{\circ}$, which gives

$$
\tau=\sigma_{1}\left\{\frac{(1-R)(1-\mu)}{2}-R \mu\right\} .
$$

The failure stress, $\sigma_{1 \mathrm{f}}$, may then be written as

$$
\sigma_{1 \mathrm{f}}=\frac{2 \tau_{\mathrm{f}}}{(1-R)(1-\mu)-2 R \mu}
$$

where $\tau_{\mathrm{f}}$ is the value of $\tau$ at failure. Since the condition for failure is not known exactly, other than that it is based upon the mode-I growth of the wings and probably wing crack interaction, $\tau_{\mathrm{f}}$ cannot be written explicitly. The best one can do is to write

$$
\tau_{\mathrm{f}}=Z K_{\mathrm{IC}}(\pi a)^{-\frac{1}{2}}
$$

where $Z$ is a dimensionless parameter which incorporates both crack interaction under the confinement and crack length at failure, and $a$ is the half length of the cracks. As apparent from the thin sections and from the earlier work on ice (Gold, 1972; Cole, 1985; Kalifa and others, 1989; Cannon and others, 1990; Schulson, 1990) the crack size is set by the grain-size, $d$; i.e. $a=d / 2$. Thus, the failure stress may be given by the expression

$$
\sigma_{1 \mathrm{f}}=\frac{2^{3 / 2} Z K_{\mathrm{IC}}}{(\pi d)^{\frac{1}{2}}\{(1-R)(1-\mu)-2 R \mu\}}
$$

Upon substituting $K_{\mathrm{IC}}=0.1 \mathrm{MPa} \mathrm{m}^{\frac{1}{2}}$ (Nixon and Schulson, 1987) and $\mu=0.75$ and, using the present data for $\sigma_{\text {lf }}=7.5 \mathrm{MPa}$ at $R=0$, Equation (6) indicates that $Z$ 
is about unity. This compares with a value closer to 2 from an earlier analysis of strength under uniaxial loading (Schulson, 1990). The failure stress (in MPa) for the conditions of the present experiment may then be given by the relationship $\left(0 \leq R<R_{0}\right)$

$$
\sigma_{1 \mathrm{f}}=\frac{1.9}{(1-R)(1-\mu)-2 R \mu} .
$$

Equation (7) is superimposed upon the plot in Figure 2, again using $\mu=0.75$. The superposition indicates fair agreement between theory and experiment within the low confinement regime. A separate plot, incidentally, of $\sigma_{1 \mathrm{f}}^{-1}$ versus $R(\leq 0.10)$ for the data in Figure 2 yielded a curve with positive curvature. This implies that $\mu$ may have decreased with increasing $R$.

In the above analysis, the value taken for $\mu$ was 0.75 from measurements under low normal stresses $(17 \mathrm{kPa})$ by Jones and others (in press) of the kinetic coefficient of friction of fresh-water ice on fresh-water ice at $-40^{\circ} \mathrm{C}$ over a range of sliding velocities. The particular velocity, $v$, for which $\mu=0.75$ corresponds is $1.5 \times 10^{-5} \mathrm{~m} \mathrm{~s}^{-1}$ and was obtained from the relationship $v \approx 2 \dot{\epsilon} d / N$ (which, for the sake of argument, assumes that the elastic, the anelastic and the viscous contributions to strain are negligible with respect to the crack sliding contribution) where $N$ is the number of cracks sliding simultaneously, taken for simplicity as one, and where $\dot{\epsilon}=10^{-3} \mathrm{~s}^{-1}$ and $d=7.3 \mathrm{~mm}$.

One other point is worth noting. The model predicts that under the loading conditions $\sigma_{2}=\sigma_{3}=R \sigma_{1}$ the degree of confinement, $R_{0}$, above which the tendency for crack sliding is zero (i.e. $\tau=0$ ) is given (from Equation (3)) by the expression

$$
R_{0}=(1-\mu) /(1+\mu)
$$

The same expression was given by Cooksley (1984). At $-40^{\circ} \mathrm{C}$, where $\mu=0.75$, this predicts $R_{0}=0.14$, in reasonable agreement with the transition between the two regimes.

An interesting outcome from this analysis is the role of the friction coefficient. Both the sensitivity of the fracture stress to confinement and the transition from one regime to the other appear to depend rather sensitively upon this property. If so, then this dependence, when coupled with the fact that $\mu$ decreases with increasing temperature (Barnes and others, 1971; Jones and others, in press), implies that with increasing temperature the sensitivity to confinement will be lower but that the range of confinement pertaining to the first regime will be greater. Experiments to test these expectations are now in progress.

Thus, the effect of relatively moderate levels of confinement on the brittle compressive fracture of ice can be understood within the context of the frictional crack sliding-wing crack mechanism. The present study, then, extends the validity of this mechanism from the fracture of ice under uniaxial loading, as discussed earlier (Schulson, 1990), to fracture under multiaxial loading.

\section{SUMMARY AND CONCLUSIONS}

A study has been made of the effect of confinement on the brittle compressive failure of coarsely grained, freshwater ice at $-40^{\circ} \mathrm{C}$. Proportional loading was applied using a true multiaxial loading system. The stress state was $\sigma_{2}=\sigma_{3}=R \sigma_{1}$ where $\sigma_{1}, \sigma_{2}$ and $\sigma_{3}$ are the principal stresses and $R$ is the degree of confinement. It is concluded that:

(1) Two regimes exist. For low levels of confinement, the fracture stress rises sharply with confinement. For higher levels, the fracture stress still rises, but less sharply. The transition occurs around $R=0.15$.

(2) Failure within the lower regime can be understood within the context of the frictional crack sliding-wing crack mechanism. Accordingly, the fracture stress can be expressed by the relationship (for $0 \leq R<R_{0}$ )

$$
\sigma_{1 \mathrm{f}}=2^{3 / 2} Z K_{\mathrm{IC}} /\left\{(\pi d)^{1 / 2}\{(1-R)(1-\mu)-2 R \mu\}\right\},
$$

where $K_{\mathrm{IC}}$ is the fracture toughness, $\mu$ is the friction coefficient, $d$ is grain-size and $Z$ is a dimensionless parameter of order unity.

(3) The transition from one regime to the other appears to occur when crack sliding can no longer occur. Accordingly, the transition may be expressed through the relationship

$$
R_{0}=(1-\mu) /(1+\mu) .
$$

\section{ACKNOWLEDGEMENTS}

The authors wish to thank Mr Douglas Fifolt for developing the data acquisition system and Dr J. Zhang, Prof. H.J. Frost, Visiting Profs. V.F. Petrenko and R.W. Whitworth for discussions. The work was supported by the Office of Naval Research, grant no. N00014-86K0695 and was performed in the Ice Research Laboratory of Thayer School of Engineering. The laboratory is operated through the additional support of the Army Research Office, Minerals Management Service, U.S. Coast Guard, Amoco, Chevron, Conoco, Exxon, Mobil, Shell and Unocal.

\section{REFERENCES}

Argon, A.S., and 7 others. 1983. Fracture in compression of brittle solids. Report NMAB-404. Washington, DC, National Academy Press.

Ashby, M.F. and S.D. Hallam. 1986. The failure of brittle solids containing small cracks under compressive stress states. Acta Metall., 34, 497-510.

Barnes, P., D. Tabor, and J.C.F. Walker. 1971. The friction and creep of polycrystalline ice. Proc. R. Soc. London, Ser. A, 324(1557), 127-155.

Cannon, N.P., E.M. Schulson, T.R. Smith, and H.J. Frost. 1990. Wing cracks and brittle compressive fracture. Acta Metall. Materialia, 38, 1955-1962.

Cole, D.M. 1979. Preparation of polycrystalline ice specimens for laboratory experiments. Cold Reg. Sci. Technol., 1(2), 153-159.

Cole, D.M. 1986. Effect of grain size on the internal fracturing of polycrystalline ice. CRREL Rep. 86-5.

Cooksley, S.D. Unpublished. Yield and fracture surfaces of brittle solids under multiaxial loading. (Ph.D. thesis, University of Cambridge, 1984.) 
Gies, M.C. Unpublished. The development of brush-type platens for loading ice in compression. (M.E. thesis, Dartmouth College. Thayer School of Engineering, 1988.)

Gold, L.W. 1972. The process of failure of columnargrained ice. Philos. Mag., 26(2), 311-328.

Griffith, A.A. 1924. Theory of rupture. Applied Mechanics, 1, 55-63.

Häusler, F.U. 1982. Multiaxial compressive strength tests on saline ice with brush-type platens. In International Association for Hydraulic Research. International Symposium on Ice, Québec, ... 1981. Proceedings. Vol. 2. Québec, Université Laval, 526536.

Jones, D.E., F.E. Kennedy and E.M. Schulson. In press. The kinetic coefficient of fresh-water ice against itself. J. Glaciol.

Jones, S.J. 1982. The confined compressive strength of polycrystalline ice. J. Glaciol., 28(98), 171-177.

Kalifa, P., P. Duval, and M. Ricard. 1989. Crack nucleation in polycrystalline ice under compressive stress states. Proceedings of the Eighth International Conference on Offshore Mechanics and Arctic Engineering ... The Hague, The Netherlands, March 19-23, 1989. Vol. IV. Arctic and polar technology. New York, American Society of Me- chanical Engineers, 13-21.

Lasonde, G.J. Unpublished. The effects of end constraint on the compressive fracture mode of polycrystalline ice in compression. (M.E. thesis, Dartmouth College. Thayer School of Engineering, 1988.)

McClintock, F.A. and J.B. Walsh. 1962. Friction on Griffith cracks in rocks under pressure. Proc. 4th U.S. Nat'l Cong. Appl. Mech., ASME, 2, 1015-1021.

Nemat-Nasser, S. and H. Horii. 1982. Compressioninduced nonplanar crack extension with application to splitting, exfoliation, and rockburst. J. Geophys. Res., 87(B8), 6805-6821.

Nixon, W.A. and E.M. Schulson. 1987. A micromechanical view of the fracture toughness of ice. J. Phys. (Paris), 48, Colloq. C1, 313-319. (Supplément au 3.)

Schulson, E.M. 1990. The brittle compressive fracture of ice. Acta Metall. Materialia, 38, 1963-1976.

Schulson, E.M., M.C. Gies, G.J. Lasonde, and W.A. Nixon. 1989. The effect of the specimen-platen interface on internal cracking and brittle fracture of ice under compression: high-speed photography. J. Glaciol., 35(121), 378-382.

The accuracy of references in the text and in this list is the responsibility of the authors, to whom queries should be addressed. 